\title{
La autonomía como indicador del desarrollo de la personalidad: los aportes de P. Ya. Galperin
}

\author{
Autonomy as an indicator of personality development: the \\ contributions of P. Ya. Galperin
}

Gloria Fariñas León ${ }^{1}$

\begin{abstract}
RESUMEN
La autonomía es uno de los indicadores centrales en el desarrollo de la personalidad (emergente del desarrollo potencial). Indicador psicológico cuyo estudio en el enfoque histórico culturalista asume características particulares pues se estudia en la dinámica de las mediaciones internas de los diferentes procesos mentales (interfuncionalidad), no de manera aislada o asociativa como en otros enfoques psicológicos. La estructura y dinámica de los cambios operados de manera dialéctica y compleja en el desarrollo, le otorgan un significado diferente porque obedecen a determinadas leyes que expresan las interrelacioes dinámicas entre las condiciones externas e internas de ese desarrollo. El presente capítulo pretende analizar las diferentes mediaciones entre los procesos (y sus indicadores fundamentales), que dan origen a la autonomía, partiendo de los aportes de la teoría de Galperin relativos a la formación de las acciones mentales; asimismo, se abordan especialmente las posibles aplicaciones con miras al diseño de la enseñanza.
\end{abstract}

Palavras-chave: Autonomia. Interfuncionalidad. Desarrollo. Personalidad.

\begin{abstract}
Autonomy is one of the central indicators in personality development (emerging from potential development). Psychological indicator whose study in the historical culturalist approach assumes particular characteristics because it is studied in the dynamics of the internal mediations of the different mental processes (interfunctionality), not in an isolated or associative way as in other psychological approaches. The structure and dynamics of the changes operated in a dialectical and complex way in development, give it a different meaning because they obey certain laws that express the dynamic interrelations between the external and internal conditions of that development. This chapter aims to analyze the different mediations between the processes (and their fundamental indicators), which give rise to autonomy, based on the contributions of Galperin's theory related to the formation of mental actions; Likewise, possible applications are addressed with a view to teaching design.
\end{abstract}

Keywords: Autonomy. Inerfunctionality. Development. Personality.

\footnotetext{
1 Professora Doutora da Universidade de La Salle del Bajío, México. ORCID: https://orcid.org/00000002-2290-3169. E-mail: glofaleon2000@gmail.com.
} 


\section{Introducción}

El estudio sobre la interfuncionalidad de los procesos mentales permanece relegado por no pocos investigadores y profesionales, aunque debiera estar en la agenda de la producción psicológica sobre todo en el campo de la educación, pues esta es condición esencial del desarrollo humano (VYGOTSKI, 2015); además, es uno de los hallazgos que muestran la profundidad del análisis complejo y dialéctico del enfoque histórico culturalista sobre la subjetividad y su desenvolvimiento durante la vida. Tenerla en cuenta, posibilita mejores análisis y soluciones para la teorización sobre la problemática de la autonomía y de otras relacionadas con dicho desarrollo; al mismo tiempo, para lograr mejores diseños y prácticas educativas. Sin embargo, esta cualidad de complejidad, a mi parecer, puede ahuyentar a todos aquellos psicólogos, cuya preferencia se oriente a enfoques simplifcadores, objetivistas o reducionistas; porque estos son más fáciles de comprender ya que frecuentemente se sustentan en la experiencia de sentido común, para la cual generalmente "más es mejor". La condición de la interfuncionalidad muestra la dudosa naturaleza de esta última aseveración y puede esclarecer diversas cuestiones cualitativas - no solo cuantitativas- en la explicación de la subjetividad en general y de la autonomía en particular.

Según esa máxima positivista, por ejemplo, es mejor un programa de enseñanza lleno de conceptos -es lo habitual- en relación con un programa cuyo foco de atención sea un método de análisis dialéctico para construir conceptos de forma integral y sistemática o histórica. La primera variante exige la evaluación de la suma de conocimientos mostrados por los estudiantes, bajo el prejuicio de "la dificultad" para valorar lo subjetivo o la falta de "rigor" en la evaluación si esto se intentara; en cambio la segunda o histórico culturalista, "se arriesga" a penetrar en la interfuncionalidad para compenderla y explicarla. La educación precisa trascender la fragmentación y la linealidad del conocimiento ocasionada por programas como el primero, con la práctica de nuevos programas dedicados a la construcción de conocimientos desde puntos de 
vista críticos sobre la realidad; máxime si estos exigen la visión total y dialéctica de esta. Este cambio necesario aunque parezca lejano a la problemática de la interfuncionalidad, está intrínsecamente ligado a ella; porque mientras en la adición de conocimientos prima la participación de la percepción y la memoria; en la elaboración de los saberes respaldada por un punto de vista crítico, sobresale el pensamiento y los restantes procesos mentales regularmente desencadenados por él (interfuncionalidad).

Los recursos psicopedagógicos concebidos para superar la fragmentación y linealidad del conocimiento de la realidad en la enseñanza, cuenta con muchos años de experimentación, en diversas formas según el objeto de análisis (GALPERIN, 1958 y otros autores histórico cultulistas después de él). Se trata de un método de análisis que demanda la intervención armónica de los diversos procesos subjetivos (o psicológicos) en el desarollo: la atención voluntaria, los procesos afectivo-motivacionales, entre otros, dirigidos por el pensamiento y el lenguaje en sus diferentes funciones, especialmente la reguladora. Este método observa la realidad en su naturaleza concatenada, por lo cual, el conjunto de saberes puede ser organizado como un todo dialéctico; lo que supera la visión de la totalidad como la suma de partes o fracciones, al mismo tiempo que supera la creencia de que "más es mejor", tan frecuente en el fundamento de la enseñanza tradicional.

La complejidad dialéctica de la realidad supone las interrelaciones históricas del todo y sus partes, a modo de concatenación universal; cualidad que un método de estudio no debiera violentar. El dominio de la percepción y la memoria en esta forma simplificadora de examinar la realidad, puede actuar por encima del funcionamiento pleno de otros procesos mentales que tienen una incidencia más directiva, como el pensamiento, la atención y el habla, en la mediación mutua o interfuncionalidad de los procesos mentales.

Cuando la enseñanza fragmenta la construcción del conocimiento -y así la participación de los procesos mentales requeridos en esta acción- con la finalidad de simplificar su dosificación, el pensamiento del estudiante retrocede en elguna forma y medida a la infancia, época caracterizada fundamentalmente 
por la percepción y la memorización, en otras palabras, por un pensamiento fundamentado en la apariencia de las cosas. En cambio, el pensamiento desarrollado (o teórico), es capaz de ir de lo abstracto a lo concreto pensado como un proceso inquisidor y penetrante en la complejidad de la realidad, donde puede encontrar las explicaciones de su esencia, más allá de lo fenoménico. Esta tarea de encontrar la verdad, exige al pensamiento la movilización de los diferentes procesos mentales; reclamo muy difícil de cumplir con enfoques positivistas fragmentadores de lo que se enseña y aprende. Fraccionamiento también causante, en alguna forma y medida, de las diversas dificultades del estudiantado para aprender y desenvolverse integralmente como personalidad; que implica una autonomía plena y estable, tema central de este análisis. Cuando el sujeto del aprendizaje, digamos en una universidad, tiende a la reproducción del conocimiento (percepción-memoria), el pensamiento no se desarrolla a escalas superiores como en el segundo caso, sigue operando en formas propias de niveles anteriores de funcionamiento mental, en consecuencia el estudiante puede no lograr la autonomía demandada por las tareas de la vida correspondientes a su edad.

\section{Autonomía y zona de desarrollo próximo}

Algunos investigadores interpretan la zona de desarrollo próximo ${ }^{2}$ como un recurso instrumental bastante "plano" (WERTSCH, 1988, entre otros autores citados por él), sin dimensiones psicólógicas esenciales a tener en cuenta en su examen; cuando la zona desarrollo próximo se caracteriza, en verdad, por el tejido dialéctico entre lo interpsíquico y lo intrapsíquico (ley genética formulada por Vygotski, 1987), durante el curso del aprendizaje-desarrollo, por consiguiente, la intención del investigador puede y debe ser más refinada en cuanto a la profundidad de sus observaciones.

En el presente trabajo propongo algunas alternativas de análisis y formas generales de intervención, para los interesados en la incursión de las

\footnotetext{
2 En lo sucesivo puede aparecer como ZDP.
} 
características de la subjetividad en desarrollo, desde la perspectiva histórico culturalista. Asumo que los lectores conocen el significado del desarrollo real o actual (significa el desarrollo potencial devenido como tal), caracterizado por la resolución autónoma o independiente de las tareas de aprendizaje, es decir, sin el apoyo imprescindible de otro sujeto.

Analizaré el entramado de procesos desencadenantes de la autonomía (indicador ${ }^{3}$ básico para el desarrollo real) a partir de las dos interrogaciones siguientes:

¿Puede ser verdaderamente autónomo un sujeto cuyo conocimiento no se haya generalizado adecuadamente?

¿Puede ser verdaderamente autónomo un sujeto cuyo conocimiento no sea crítico?

Ambas respuestas son negativas desde la óptica histórico culturalista en general y la galperiniana en particular. Agregaría que el contenido de la generalización y la criticidad dependen además, y en determinada medida, de las tareas socioculturales exigidas al sujeto en cada etapa de su desarrollo; asimismo, de la estructura dinámica de la conciencia lograda en la zona de desarrollo próximo para solucionarlas.

Pudiéramos formular estas mismas preguntas con cada uno de los indicadores (o características) de los procesos psicológicos y la respuesta sería la misma. Veamos las razones.

La autonomía se sostiene sobre la interconexión de los procesos psíquicos, según la estructura de la conciencia alcanzada gracias a la vivencia de la interrelación (actividad-comunicación) con los otros seres humanos, mediante las cuales se materializan las exigencias de cada periodo de la vida; responde a la ley genética ya mencionada que actúa en determinadas condiciones históricas y culturales, entre otras regularidades. Es concordancia con lo anterior no debiéramos tomar los periodos etarios de modo rígido, este es uno de los valores del concepto de zona de desarrollo

\footnotetext{
${ }^{3}$ Asumo los indicadores psicológicos -parámetros para Galperin- tal como este autor, es decir como cualidades o manifestaciones de la psiquis -en todos sus niveles de organización-en la solución o planteamiento de los diversos problemas o tareas de la vida, en esta oportunidad de la vida académica
} 
próximo: la personalización del análisis sobre el desenvolvimiento del sujeto, único y por consiguiente, no estandarizable en un diagnóstico del desarrollo.

\section{Una alternativa de análisis}

En varias oportunidades he argumentado (FARIÑAS, 2017, 2019a, 2019b) la necesidad de reanalizar en general la obra de diversos autores histórico culturalistas desde la perspectiva de la complejidad dialéctica (GONZÁLEZ, 2015) o de la complejidad en el caso de Morin (1999 y 2000). Sin embargo, en esta oportunidad me limitaré a destacar nuevamente la necesidad de una lectura compleja dialéctica de la obra de Galperin (Fariñas, 2019b), en lo referente a la autonomía. Es cierto que realizo una lectura entrelíneas de sus textos, pues lamento siempre la comprensión simplificadora de algunos psicólogos y pedagogos rayana con un positivismo a veces extremo. Estamos en presencia de una obra rica en generalidades y detalles aprovechables a los fines de un análisis complejo dialéctico, sobre las relaciones interdependientes entre lo interpsíquico y lo intrapsíquico (en la ZDP), al mismo tiempo de la interdependencia (interfuncionalidad) de lo intrapsíquico. Me propongo siempre un juicio ponderado -ni unilateral, ni demoledor-, y en el caso de Galperin, hay bastante que inferir tanto para el desarrollo de la teoría como de la práctica.

Analizo los aportes de la teoría de este autor y sus derivaciones metodológicas: para el diseño pedagógico -o pisopedagógico- de la zona de desarrollo próximo (unidad aprendizaje-desarrollo). Esto no significa ausencia de insatisfacciones sobre la teoría de Galperin; pudiera analizar en otra oportunidad, por ejemplo: la adecuación de su denominación, las consideraciones específicas sobre la comunicación, los momentos de cooperación en la zona de desarrollo próximo, entre otras apreciaciones. La cuestión central ahora es considerar sus aportes más notables, en relación con la conceptualización de la autonomía en situaciones de enseñanza académica, con el objetivo de contribuir en alguna medida a la sistematización del conocimiento psicológico producido por el enfoque histórico culturalista. 
El punto de vista sobre la autonomía que observo en Galperin responde las preguntas formuladas anteriormente. Es imprescindible aclarar que asumo la interpretación de la teoría de este autor en su relación con Vygotski, con el mayor apego posible a la obra escrita; quizá es mejor decir que asumo esta teoría -como otras-, con todo respeto y principalmente en su espíritu, también con el propósito de no olvidar la letra.

La autonomía, para su mejor formación, debe cumplir determinados requisitos psicológicos y educativos en su formación (FARIÑAS, 2019), pues no emerge espontáneamente gracias a la inmanencia del desarrollo. Ella es una característica secundaria de acuerdo con la comprensión galperiniana sobre los parámetros de la acción (indicadores psicológicos o cualidades en el presente capítulo). Como en la historia evolutiva de un sujeto, deben suceder diversas mediaciones entre los procesos mentales estructurantes de la conciencia para la mejor solución de las tareas propias de la vida en cada edad, la autonomía se convierte en uno de los indicadores más elocuentes del desarrollo psicológico, complejo por naturaleza, debido a la interrelación dialéctica requerida a los procesos mentales intervinientes en la zona de desarrollo próximo.

Sin necesidad de explicar todos y cada uno de los indicadores considerados por Galperin porque escribo para especialistas, es esencial recordar el papel de los diferentes procesos mentales en la interconexión propia de un buen desarrollo (válido para los indicadores de los procesos psíquicos tratados en el capítulo):

[...] cuanto sabemos acerca del desarrollo psíquico indica que consiste en la transformación de la estructura interfuncional de la conciencia. La psicología debe hacer de estas relaciones y de sus cambios evolutivos su principal problema, el núcleo de su estudio, en vez de postular meramente la interrelación general de las funciones. Este desplazamiento metodológico es imprescindible para un estudio fructífero del lenguaje y el pensamiento ${ }^{4}$ (VYGOTSKI, 2015, p. 68).

\footnotetext{
${ }^{4} \mathrm{El}$ pensamiento y el lenguaje son procesos rectores del desarrollo en las distintas edades, aun cuando su expresión esté basada en la precepción o en la memoria como es el caso de las primeras edades.
} 
Y más adelante plantea: "Si para la antigua psicología el campo de las relaciones interfuncionales era inaccesible a la investigación, ahora, en cambio, se abre a quienes están dispuestos a emplear el método de análisis de unidades" (VYGOTSKI, 2015, p. 75).

Asimismo, enfatiza:

Se ha demostrado con pruebas experimentales que el desaarrollo mental no coincide con el desarrollo de las funciones psicológicas aisladas, sino que depende más bien de las relaciones cambiantes entre ellas. El desarrollo de cada función, a su vez, depende del progreso en el desarrollo del sistema interfuncional. La conciencia al desarrollarse como un auténtico todo, cambia su estrutura interna en cada nueva etapa. Así pues, el destino de cada componente funcional de la conciencia depende del desarrollo del sistema entero.

[...] las relaciones interfuncionales, lejos de ser inmutables, experimentan un desarrollo considerable. Además, este desarrollo, es decir, los cambios en la composición funcional de la conciencia, son el verdadero núcleo del desarrollo (VYGOTSKI, 2015, p. 233).

Asumiré como unidad de análisis, el cambio de esta composición interfuncional de la conciencia (supone las mediaciones entre los procesos y su expresión mediante indicadores) en la conformación de la autonomía.

Más adelante apunta:

[...] lo central para toda estructura de la conciencia y para todo el sistema de actividad de las funciones psíquicas lo constituye el desarrollo del pensamiento. Con ello guarda también relación la idea de la intelectualización de todas las funciones restantes, es decir, sus variaciones dependen de que en una determinada fase del pensamiento lleva a la atribución del sentido de esas funciones que el niño comienza a comportarse racionalmente hacia su actividad psíquica. Debido a ello toda una serie de funciones que actuaban automáticamente comienzan a hacerlo consciente, lógicamente. Aún mayor importancia tiene el que de aquí se realizan intentos (y en ellos radica, a mi parecer, el valor de esta idea para la pedagogía) de demostrar que el grado de desarrollo de sus categorías, son las premisas psicológicas del desenvovlimiento de un determinado sistema de pensamiento infantil consciente o no consciente [...] y la realidad exterior, su dependencia del aspecto semántico del lenguaje infantil (VYGOTSKI, 2015, p. 400).

A partir del análisis de estas citas, presto atención a lo siguiente en relación con la autonomía: 
a) Al ser el indicador (o cualidad) esencial del desarrollo real o actual (VYGOTSKI, 1978, 2015), tiene el peso suficiente para ser analizada en particular, porque el desarrollo es centro de observación en el enfoque histórico culturalista. Sería inconsecuente abordarla grosso modo o abstractamente, así se limitaría el alcance del análisis y la aplicación en el plano específico (pedagógico, familiar, etcétera), por tanto la analizo concretamente como resultado de la interrelación específica entre los procesos psicológics, razón por la cual "desmenuzo" su composición, sin observarla como la adición de procesos-indicadores, sino como la urdimbre dinámica de estos.

b) Es necesario tener en cuenta el papel del pensamiento en el logro de la autonomía en cada momento del desarrollo, de acuerdo con la estructura de la conciencia dentro de la cual opera. Significa la interrelación pensamiento-lenguaje-atención en la resolución autónoma de las diferentes tareas de aprendizaje. Esta es una manera de concretar las exigencias psicológicas o psicopedagógicas del diseño y realización de dichas tareas, siempre concebidas en forma de sistema para retar y abarcar los procesos mentales imprescindibles en la fragua del desarrollo. Es decir, esos procesos al requerir mediación mutua, no fragmentación, deben ser motivados por un diseño sistémico, no por una recopilación cualquiera de tareas o problemas a resolver.

c) $\mathrm{Si}$ el desarrollo mental depende de la interfuncionalidad, y no de funciones psicológicas aisladas, la autonomía debe ser analizada como un indicador complejo dialéctico de la conciencia, expresión del funcionamiento del todo, nos refiramos a este como sujeto del desarrollo o como personalidad. El sujeto al tener la posibilidad de autodirigirse y autorregularse, no requiere constantemente de la ayuda de los demás para la resolución de las tareas.

Seleccioné una última cita de Vygotski sobre la atención, con la finalidad de fundamentar el análisis acerca de la autonomía como indicador del sistema de autorregulación, alcanzado por el sujeto en correspondencia con las formas de 
estructuración de la conciencia a través de vida. Ella nos permitirá comprender mejor los planteamientos de Galperin sobre estas cuestiones:

Si tenemos en cuenta que la atención es una función de estructuración de lo percibido y representado por la memoria resulta fácil comprender que ya en el umbral de la edad escolar el niño goza de una atención y una memoria suficientmente maduras. Dispone, por consiguiente, de lo que debe tomar conciencia y de lo que debe dominar. Resulta comprensible por qué las funciones conscientes y voluntarias de la memoria y la atención son centrales en esa edad.

Hasta entonces, el niño piensa en ideas generales o complejos, tal como hemos denominado en otro lugar esta estructura más temprana de generalización predominante en la edad preescolar (VYGOTSKI, 2015, p. 211).

Trato de completar el análisis con el siguiente planteamiento de Galperin, ya que la autonomía también resulta, en última instancia, de la función estructuradora de la atención, subyacente en el sistema de regulación del sujeto. Estos son los hallazgos a resaltar:

[...] la atención interna ${ }^{5}$ se forma del control del contenido objetal de la acción [...] exige la confrontación de la tarea con su ejecución. Por consiguiente, el control constituye una parte esencial e imprescindible de ese manejo. [...] el control constituye un elemento integrante en la psiquis como actividad de orientación ${ }^{6}$. [...] en un acto en que "el yo" se dirige a ese contenido. [...] Sin embargo, a diferencia de otras acciones, [...] la actividad de control no tiene un producto independiente. Ella siempre va dirigida a aquello que, aunque sea parcialmente, ya se ha creado por otros procesos, es decir, para controlar hay que tener qué controlar (GALPERIN, 1973, p.22).

Esos actos de dirección del yo, gracias a la atención interna, pueden regular los procesos mentales y su interfuncionalidad, base de la autonomía. El foco de observación sería la unidad indisoluble pensamiento-lenguaje-atención, pues supone la participación de los diferente procesos mentales; no por gusto Vygotski (2015) le dedicó todo un libro al análisis de los posibles postulados y

\footnotetext{
${ }^{5}$ No podemos perder de vista, que el lenguaje interno es el basamento de la atención interna y a la vez del pensamiento, entre otros procesos. El lenguaje interno anida las distintas funciones del lenguaje, por tanto, se puede transformar en lenguaje externo para los otros (comunicación) o ejercer su función reguladora sobre la atención, el pensamiento y los otros procesos psicológicos. ${ }_{6}^{6}$ Galperin concibe la psiquis como actividad de orientación (1979).
} 
los experimentos sobre esta problemática que dio fundamento al papel de la interfuncionalidad en la zona de desarrollo próximo. El lenguaje interno sustenta la atención interna y el pensamiento, por consiguiente, el modo autorregulado de funcionar.

A propósito de este destaque y para no dejar confusa la unidad entre lo cognitivo y lo afectivo en este análisis, baste citar otra conclusión muy elocuente:

El propio pensamiento no toma origen en otro pensamiento, sino en la esfera motivacional de nuestra conciencia, la que abarca nuestros deseos y necesidades, nuestros intereses y motivos, nuestros afectos y emociones. Tras el pensamiento se encuentra una tendencia afectiva y volitiva, la única que puede dar respuesta al único 'por qué' en el análisis del pensamiento (VYGOTSKI, 2015, p. 2009).

a) La autonomía como indicador central del desarrollo real: $1^{\circ}$ depende de la dirección de la atención interna (devenida sistema de regulación) en la interrelación del sujeto con los otros. $2^{\circ}$ expresa el nivel o calidad de la interfuncionalidad de las funciones mentales regidas por el pensamiento, de acuerdo con la estructura de la conciencia del sujeto en cada momento del desarrollo. $3^{\circ}$ para mejores consecuencias, se sostiene en el lenguaje interno (lenguaje intelectual) en su íntima ligazón con el pensamiento (pensamiento verbal). En otras palabras, como cualidad proviene de los logros de la atención interna en el aglutinamiento complejo y dialéctico de los procesos mentales dirigido por el pensamiento-lengiaje, en la estructura de la conciencia.

b) La autonomía como macrocaracterística ${ }^{7}$ puede tener diversa calidad según haya cristalizado la mediación entre los procesos mentales y sus características o indicadores (generalización, racionalidad, concientización, criticidad, creatividad, responsabilidad, etcétera, planteadas a continuación).

\footnotetext{
7 En mi interpretación, la autonomía desde la perspectiva galperiniana, es una cualidad (indicador) macroscópica en la acción reguladora de la personalidad, donde la unidad dialéctica pensamiento-lenguaje-atención es regente de los procesos del desarrollo. No olvidemos el llamado lenguaje de autor (BAKHTIN, 2009), una de las mejores expresiones de la autonomía.
} 


\section{Sobre los indicadores psicólógicos de los procesos en la interfuncionalidad de la conciencia}

Valoraré estas dinámicas con el apoyo de mis lecturas sobre la teoría de Galperin, mi práctica investigativa y la práctica docente diaria. Tomaré como centro de atención los indicadores psicológicos, que según este autor expresan un desarrollo apropiado, cuando estos se median entre sí para solucionar los problemas y tareas de aprendizaje.

a) La generalización y la racionalidad expresan la construcción de un conocimiento (conceptos, valores, habilidades, etcétera) adecuado y aplicable en distintas situaciones (transferencia); mas es necesario aclarar, que la racionalidad matiza el tipo de generalización. Hay que diferenciar la racionalidad positivista que fragmenta, simplifica $y$ establece relaciones lineales y externas ${ }^{8}$ en la construcción del conocimiento, de la racionalidad compleja dialéctica orientada al establecimiento de la esencia en los nexos históricos intrínsecos que concatenan los fenómenos y procesos de la realidad en estudio (GALPERIN, 1958; DAVIDOV, 19819). Quiere decir, dos modos fundamentales de pensar: el primero, está restringido a determinados campos de la acción por su posición reduccionista y unilateral ante una realidad esencialmente compleja y dialéctica; el segundo, se adecua a las complejidades de un universo cambiante, en el cual es necesario descubrir las raíces históricas de los fenómenos y procesos de la relidad como totalidad -en sus nexos intrínsecos-, para influir apropiadamente sobre estos en el contexto histórico y cultural correspondiente. La primera visión tiende al estatismo que violenta las leyes de la realidad en desarrollo; la

\footnotetext{
8 Por ejemplo, las asociaciones por contigüidad y semejanza en la memoria y las conexiones estímulo-respuesta en el comportamiento, que acontecen y son accedidas mediante el análisis fenoménico, que no es equivalente a la esencia de la memoria y el comportamiento.

9 En el caso de Galperin, se refiere especialmente a la orientación tipo III, distinguida por la construcción de un método de análisis, que integra y sistematiza dialécticamente el conocimiento (lo general-lo particular, en un todo), analizado por Davidov como pensamiento teórico, que no es otra cosa que el pensamiento complejo dialéctico.
} 
segunda, se erige sobre estas a favor de la transformación apropiada de la realidad (GONZÁLEZ CASANOVA, 2015).

b) La criticidad, la concienciación y la responsabilidad (esta última más estudiada en el último periodo de trabajo del autor -y expuesta de manera más integral en su libro Introducción a la psicología (GALPERIN, 1979), atribuyen de sentido (según los valores éticos, estéticos, científicos, etcétera) a los significados construídos por el sujeto del aprendizaje en el contexto histórico cultural. Esta unidad sentido-significado se expresa en sus diversas actitudes ante esa realidad.

c) Por otra parte, la creatividad como expresión del carácter único e irrepetible de la personalidad, se puede manifiestar en los educandos desde que comenzamos a operar como docentes o investigadores en la zona de desarrollo próximo. Según Zinchenko uno interioriza lo suyo que debe crear, no lo ajeno (1990). En otras palabras, el pensamiento, o mejor la unidad pensamiento-palabra-atención, en función reguladora de la imaginación-creación, activa todo ese proceso de apropiación de la cultura y en este su originalidad, ${ }^{10}$, el sello de autoría ya mencionado cuando me referí más arriba a Bakhtin. Sin embargo, la creatividad al igual que los otros indicadores, deben estar mediados mutuamente. Desde la perspectiva histórico culturalista no sería adecuado, por ejemplo, un acto de creación si no fuera consciente, crítico y responsable; estos indicadores acotan la eticidad de lo creado.

En síntesis, la autonomía no debe observarse como la simple solución independiente de las tareas o problemas planteados al sujeto del aprendizaje, al margen de su dinámica de formación; requieren ser analizados los matices de su forja en el establlecimicneto de la interfuncionalidad de los procesos e indicadores psicológicos que la manifiesten. Entre otros ejemplos posibles, la criticidad es expresión de un pensamiento (y lenguaje) apoyado en la esfera afectivo motivacional, cuya base son los valores (éticos, científicos, estéticos, entre otros). Muestra de que es la unidad de lo cognitivo y lo afectivo (vivencia),

10 También se considera la flexibilidad del pensamiento, aunque no la abordaré en esta oportunidad. 
la que puede poner la primera piedra de la interfuncionalidad, refinada por los procesos e indicadores mencionados; lástima entonces de la educación fundamentada en el positivismo que fracciona esa unidad, obstaculizando de una forma u otra la mediación entre los procesos requerida en el desarrollo.

Por una parte, la adecuación del conocimiento construido, es decir, la comprensión de la esencia del objeto de estudio y su transferencia a las diferentes situaciones de la vida, y por otra, la postura personal crítica y creativa ante estas, apoyan la formación del carácter en la construcción dinámica y compleja de los sentidos y saberes, mejor garante del desarrollo integral de la personalidad.

Existen otros indicadores que manifiestan, por ejemplo, la estabilidad (hábito), desenvoltura y dominio con que actúa el sujeto ante el planteamiento y solución de los problemas y tareas de la vida (como ya dije, en esta oportundiad la académica), sobre la base de la mediación entre los procesos psicológicos. Estos son los indicadores de: forma (mental o de lenguaje interno, de lenguaje externo, de operatoria material, entre otras). La automatización y la solidez (también expresiones del hábito). Estimo que las consideraciones planteadas son suficientes para darse cuenta de la magnitud de la autonomía, por aglutinar los diferentes indicadores de los procesos mentales; con lo cual destaco nuevamente la exigencia de observarla con profundidad, no grosso modo. En teoría, todos los procesos mentales debieran desarrollarse al tope, mostranto también al tope el tejido de sus indicadores, pero esto verdaderamente no funciona así. La didáctica derivada del enfoque histórico culturalista, no es una panacea y su interpretación simplificada puede constituirse en un boomerang que golpee negativamente la concepción y la práctica, haciéndoles perder credibilidad; hay que concebirla de manera compleja dialéctica, no caracterizada por la sincronía y el progreso lineal de los procesos del desarrollo de la personalidad (VYGOTSKI, 1987). 


\section{La arista social de estas valoraciones psicológicas}

Este tejido de procesos mentales (y sus indicadores) constituye el fundamento de una autonomía responsable construída en cooperación, no la comprensión liberal de la autonomía, como puede apreciarce en tendencias como las humanistas nortemericanas que enfatizan la libertad de creación responsable principalmente sobre la base de la empatía hacia el otro, no propiamente de la cooperación, la dignidad del otro y de sí mismo. Por su parte, el llamado enfoque de "las competencias», abona la visión fraccionada de los procesos piscológicos, por ende del sujeto y su personalidad, con un fin básicamente instrumental. Sucede de manera similar en el enfoque sobre el «emprendedurismo» orientado con el mismo fin a la creación de empresas y su eficiencia, no al desarrollo de la personalidad de los ciudadanos. Tanto el enfoque de las competencias como este últimio obedecen a las posiciones neoliberales del Banco mundial y de organizaciones, que lo apoyan en el terreno de la educación como Tuning para América Latina, entre otras.

Entiendo que para un autor histórico culturalista, la autonomía de un sujeto nace en la cooperación y debe operar por el bien social común (para la cooperación). Por otra parte, la conciencia y la criticidad que la median deben fundamentarse en el conocimiento profundo, no enajenado, no colonizado de la realidad.

Como dice Marcuse:

[...] Y es esta solidaridad la que ha sido quebrada por la productividad integradora del capitalismo y por el poder absoluto de su máquina de propaganda, de publicidad y de administración. Es preciso despertar y organizar la solidaridad en tanto necesidad biológica de mantenerse unido contra la brutalidad y la explotación inhumanas. Esta es la tarea. Comienza con la educación de la conciencia, el saber, la observación y el sentimiento que aprehende lo que sucede: el crimen contra la humanidad. La justificación del trabajo intelectual reside en esta tarea, y hoy el trabajo intelectual necesita ser justificado» (MARCUSE, 2016, p. 26).

Y más adelante especifica: 
[...] nos encontramos ante uno de los aspectos más perturbadores de la sociedad industrial avanzada: el carácter racional de su irracionalidad. Su productividad y eficiencia, su capacidad de incrementar y difundir sus comodidades, de convertir lo superfluo en necesidad y la destrucción en construcción, el grado en que esta civilización transforma el mundo-objeto en extensión de la mente y el cuerpo...La gente se reconoce en sus mercancías; encuentra su alma en su automóvil, en su aparato de alta fidelidad, su casa, su equipo de cocina. El mecanismo que une el individuo a su sociedad ha cambiado, y el control social se ha incrustado en las nuevas necesidades que ha producido» (MARCUSE, 2016, p. 47).

En otras palabras, la conciencia, para ser crítica y responsable necesita desprenderse críticamente de lo que él llama incrustaciones. También señala:

Si el trabajador y su jefe se divierten con el mismo programa de televisión y visitan los mismos lugares de recreo, si la mecanógrafa se viste tan elegantemente como la hija de su jefe, si el negro tiene un Cadillac, si todos leen el mismo periódico, esta asimilación indica no la desaparición de las clases, sino la medida en que las necesidades y satisfacciones que sirven para la preservación "del sistema establecido" son compartidos por la población subyacente». (MARCUSE, 2016, p. 47).

El sujeto no es incauto al pensar -por ende, tampoco el investigador o el profesional, los valores orientan su parecer. La unidad pensamiento-habla en su función reguladora tanto en el análisis consciente y crítico como en el acto de creación se funda en ellos (motivaciones para Gouveia, 2005); es esta la forma en que dicha unidad incide sobre los restantes procesos en mediación, marcando el estilo peculiar del sujeto (o personalidad). Recordemos que Galperin (1979) considera dos niveles de orientación y regulación (autorregulación) en el individuo: los procesos (sujeto) y la personalidad; a los que integra la dimensión de la persona, o sea, la dimensión valoral, la responsabilidad.

\section{El diseño didáctico}

¿Qué valor tiene todo lo examinado más arriba para el diseño de la enseñanza? 
Los sistemas educacionales que preservan los métodos conservadores de trabajo, están acostumbrados a analizar grosso modo los resultados del aprendizaje, no las características de los procesos tras estos, que profundizan el entendimiento del aprendizaje en la conducción del desarrollo (aprendizajedesarrollo). Esas concepciones y prácticas tradicionales obstaculizan de algún modo la solución de muchas de las dificultades del desenvolvimiento académico del alumnado -y por consiguiente la vida social e íntima de este- datadas por siglos, aunque hayan cambiado de apariencia.

Una de las características disitintivas del enfoque histórico culturalista consiste en lo contrario: ponderar los procesos mentales y su cristalización en los resultados del aprendizaje; en otros términos, este punto de vista permite transparentar los sucesos psicológicos en la zona de desarrollo próximo. Y esa clarificación se puede realizar mediante un sistema de tareas de aprendizaje y su control-retroalimentación en las distintas asignaturas, es así que se pueden captar y especificar los avances, detenciones, retrocesos de los estudiantes con la finalidad de orientarlos en los alcances del desenvolvimiento multilateral e integral de la personalidad.

Los indicadores que muestran las interrelaciones entre los procesos psicológicos, estudiados de una u otra manera por Galperin $(1958,1973)$ y colaboradores como Talizina (1988, 1998), juegan un papel crucial en tal clarificación ya que detallan cualitativamente lo alcanzado y por alcanzar en el desarrollo. Solo así, la educación puede incidir con mayor adecuación y oportunamente en la dinámica de la zona de desarrollo próximo, cuya naturaleza revela la participación de los procesos mentales requeridos para un mejor desarrollo integral del estudiantado; sin que esto signifique ser una panacea dudáctica.

Sigo la lógica anterior en el análisis de los indicadores para visualizar vías generales de atención a la dinámica aprendizaje-desarrollo:

a) Como observamos, no puede tomarse a la ligera la autonomía del estudiantado porque no se trata, como dije anteriormente, de cualquier tipo de autonomía. Se requiere atender con cuidado la elaboración de un 
sistema de tareas que dinamice intencionadamente la zona de desarrollo próximo; no se trata solo de garantizar un clima de trabajo en cooperación, sino también las tareas autónomas dentro y fuera del trabajo conjunto. Según Bleger (1961), en referencia a este aspecto, el trabajo en un grupo operativo debe lograr la mayor homogeneidad posible en la tarea a resolver, con la mayor hetrrogenidad en su abordaje o solución; es decir, la tarea aglutina a los estudiantes y posibilita la cooperación al mismo tiempo que la propuesta personal (única) para la resolución de la tarea grupal. No podemos olvidar la arista ética del trabajo colectivo o individual: el bien social común.

b) Las tareas deben propiciar la participación de los procesos mentales en su interrelación dinámica. Supone tener en cuenta los indicadores vistos para la potenciación del desarrollo., y dentro de estos velar la generalización de racionalidad compleja dialéctica, de modo consciente y crítico; al mismo tiempo su estabilidad. Esta conformación de la autonomía potencia mejor el desarrollo integral del sujeto y su personalidad, en comparación con la racionalidad reduccionista y fragmentaria.

\section{Conclusiones}

El diseño y realización de la educación para ser esencialmente innovador, precisa superar el paradigma de linealidad propios del conductismo y otros enfoques orientados a los resultados inmediatos del aprendizaje, con el desconocimiento de los procesos mentales que actúan o deben actuar mediándose entre sí para propiciar el desarrollo. El cambio de paradigma es imprescindible para resolver los problemas tradicionales de la enseñanza en la formación del estudiantado, no se trata meramente de modernizarla con tecnología, hay que atender la manera en que el estudiante piensa y actúa a fin de no utilizar la técnica de manera fútil. El psicólogo debe ser un aliado del docente y saber dialogar con este para el cambio de la educación. La pedagogía está urgida de una mayor comprensión psicológica, con la finalidad de un mejor 
fundamento científico de la docencia. Y aunque ningún enfoque psicológico es una receta mágica, el enfoque histórico culturtalista ha mostrado vías más específicas para incidir positivamente sobre el desarrollo del educando.

Ninguna experiencia que se precie de vanguardia, puede ignorar la importancia del desarrollo del educando como un sujeto autónomo y responsable; y el aprendizaje memorístico no es un buen sustento para propiciarlo. La autonomía no se forma directa y progresivamente estudiando conceptos, en consecuencia, hay que asumir su complejidad para no violentar su estructura y dinámica. Galperin profundiza y detalla atentamente lo que sucede en la zona de dessarrollo próximo, cuando el aprendizaje provoca la participación de los procesos mentales, especialmente del pensamiento-habl en sus disitntos aniveles de realización; asimismo, la atención como control de los procesos y resultados porque los orienta y regula en el establecimiento de su interdependencia.

No quiere decir descartar a la memoria como un proceso básico en el desarrollo, porque es esta la que atesora los cambios sustanciales del desenvolvimiento humano. Preservar los contenidos del aprendizaje de manera abstracta y mecánica, como estimula la educación tradicional, es bien distinto a preservarlos orgánicamente en forma dialéctica. No se trata de emplear recursos mnemotécnicos para pensar, sino de pensar de manera racional (en la comprensión galperiniana), crítica y creativa.

Finalmente pienso que debemos respetar el legado científico histórico culturalista, no para repetirlo y convertirlo en dogma. Es fundamental que lo veamos desde distintas ópticas, siempre complejas y dialécticas en las condiciones históricas y culturales en que vivamos; esto también preservaría su espíritu. Quizá el análisis presentado aquí en algunos momentos no se ajuste a la letra explícita del autor, porque he tratado de exponer las ideas de acuerdo con mi experiencia como investigadora y docente, no solo de la lectura de la teoría. 


\section{Referencias}

BAKHTIN, $\mathrm{M}$. El autor y el héroe en la actividad estética. Criterios, La Habana, n. 31, p. 109-130, enero-junio. 2009.

BLEGER, J. Grupos operativos en la enseñanza. En: Conferencia de la Asociación Argentina de Psicología y Psicoterapia de Grupo, Anales. 1961. Buenos Aires: 1961. p.12-30.

DAVIDOV, V. Tipos de generalización en la enseñanza. La Habana: Pueblo y Educación, 1981. 220 p.

FARIÑAS, G. Aprendizaje y desarrollo desde la perspectiva de la complejidad. México: Parmenia y La Habana: Félix Varela, 2017.120 p.

FARIÑAS, G. Aprendizaje y desarrollo desde la perspectiva de la complejidad. La teoría en la práctica. La Habana: Félix Varela, 2019a. 145p.

FARIÑAS, G. Galperin revisitado desde el pensamiento complejo (autoorganización del aprendizaje y desarrollo humano), en: Linhas críticas, Uberlândia, n. 24, 2019b. p. 284-301. https://doi.org/10.26512/lc.v24i0.20243

GALPERIN, P. "Tipos de orientación y tipos de formación de las acciones y conceptos", en Informes de la ACP de la RSFSR, no. 2, 1958. p. 13-17.

GALPERIN, P. Teoría de la formación por etapas de las acciones mentales: seis conferencias. Traducción y edición de Martínez G. La Habana: Ediciones Ligeras Universidad de La Habana, 1973. 30p.

GALPERIN, P. Introducción a la psicología. Madrid: Visor. 130p.

GONZÁLEZ, P. De la sociología del poder a la sociología de la explotación: pensar América Latina en el siglo XXI. México: Siglo XXI Editores, 2015. 192p.

GOUVEIA, V. A natureza motivacional dos valores humanos: Evidências acerca de uma nova tipología. Estudos de Psicologia, Natal, n.8, p. 431-443, 2003. https://doi.org/10.1590/s1413-294x2003000300010

MARCUSE, H. El hombre unidimensional. Barcelona: Planeta, 2016. 202 p.

MORIN, E. y Le Moigne. A inteligência da complexidade. Sao Paulo: Peirópolis, 1999. 196p.

MORIN, E. A cabeça bem feita. Repensar a reforma, reformar o pensamento. Río de Janeiro: Bertrand, 2000. 215p. 
TALIZINA, N. Psicología de la enseñanza. Moscú: Progreso, 1988. 180p.

TALIZINA, N. Pedagogicheskaya psickhologia. Moskba: Akademia, 1998. 187p.

VYGOTSKI, L. Interaction between learning and development. In: GAUVIN, M.; COLE, M. Readings on the development of Children. New York: SCIENTIFIC AMERICAN BOOKS, 1978, P. 34-40.

VYGOTSKI, L. Historia del desarrollo de las funciones psíquicas superiores. La Habana: Científico-Técnica, 1987. 193 p.

VYGOTSKI, L. Pensamiento y lenguaje. México: Paidós, Booket, 2015. 490 p.

WERTSCH, J. Vygotsky y la formación social de la mente. México: Paidós, 1988. 193p.

ZINCHENKO, V. Entrevista. En: Shuare, M. La psicología soviética tal como yo la veo. Moscú: Progreso, 1990. 159p.

Recebido em abril 2020. Aprovado em julho 2020. 